\title{
Longitudinal Screening Detects Cognitive Stability and Behavioral Deterioration in ALS Patients
}

\author{
Susan Woolley $\mathbb{D}^{1},{ }^{1}$ Ray Goetz, ${ }^{2}$ Pam Factor-Litvak $\left(\mathbb{D},{ }^{3}\right.$ Jennifer Murphy, ${ }^{4}$ Jonathan Hupf, ${ }^{5}$ \\ Catherine Lomen-Hoerth, ${ }^{6}$ Howard Andrews, ${ }^{7}$ Daragh Heitzman, ${ }^{8}$ Richard Bedlack, ${ }^{9}$ \\ Jonathan Katz, ${ }^{1}$ Richard Barohn, ${ }^{10}$ Eric Sorenson, ${ }^{11}$ Bjorn Oskarsson, ${ }^{12}$ \\ Americo Fernandes Filho, ${ }^{13}$ Edward Kasarskis, ${ }^{14}$ Tahseen Mozaffar, ${ }^{15}$ Sharon Nations, ${ }^{16}$ \\ Andrea Swenson, ${ }^{17}$ Agnes Koczon-Jaremko, ${ }^{18}$ Georgia Christodoulou, ${ }^{5}$ \\ and Hiroshi Mitsumoto ${ }^{5}$
}

${ }^{1}$ Department of Neurosciences, Forbes Norris ALS Research Center, Sutter Pacific Medical Foundation, USA

${ }^{2}$ Department of Psychiatry, New York State Psychiatric Institute, Columbia University Medical Center (CUMC), USA

${ }^{3}$ Department of Epidemiology, CUMC, Mailman School of Public Health, USA

${ }^{4}$ Department of Neurology, University of California, San Francisco (UCSF), USA

${ }^{5}$ Department of Neurology, Eleanor and Lou Gehrig MD/ALS Research Center, CUMC, USA

${ }^{6}$ Department of Neurology, UCSF, USA

${ }^{7}$ Departments of Biostatistics and Psychiatry, CUMC, Mailman School of Medicine, USA

${ }^{8}$ Texas Neurology, PA, USA

${ }^{9}$ Duke University, USA

${ }^{10}$ Department of Neurology, University of Kansas, USA

${ }^{11}$ Mayo Clinic, Rochester, USA

${ }^{12}$ University of California, Davis, Mayo Clinic, Jacksonville, USA

${ }^{13}$ University of Nebraska Medical Center, USA

${ }^{14}$ University of Kentucky, Lexington, USA

${ }^{15}$ University of California, Irvine, USA

${ }^{16}$ University of Texas, Southwestern, USA

${ }^{17}$ University of Iowa, USA

${ }^{18}$ Hospital for Special Care, USA

Correspondence should be addressed to Susan Woolley; woolles@sutterhealth.org

Received 8 April 2018; Revised 8 August 2018; Accepted 10 September 2018; Published 31 October 2018

Guest Editor: Francesca Trojsi

Copyright (c) 2018 Susan Woolley et al. This is an open access article distributed under the Creative Commons Attribution License, which permits unrestricted use, distribution, and reproduction in any medium, provided the original work is properly cited.

Objective. To evaluate longitudinal cognitive/behavioral change over 12 months in participants enrolled in the ALS Multicenter Cohort Study of Oxidative Stress (ALS COSMOS). Methods. We analyzed data from 294 ALS participants, 134 of whom were studied serially. Change over time was evaluated controlling for age, sex, symptom duration, education, race, and ethnicity. Using multiple regression, we evaluated associations among decline in ALS Functional Rating Scale-Revised (ALSFRS-R) scores, forced vital capacity (FVC), and cognitive/behavioral changes. Change in cognitive/behavioral subgroups was assessed using one-way analyses of covariance. Results. Participants with follow-up data had fewer baseline behavior problems compared to patients without follow-up data. We found significant worsening of behavior (ALS Cognitive Behavioral Screen (ALS CBS) behavioral scale, $p<0.001$; Frontal Behavioral Inventory-ALS (FBI-ALS) disinhibition subscale, $p=0.044$ ). Item analysis suggested change in frustration tolerance, insight, mental rigidity, and interests $(p<0.05)$. Changes in ALSFRS-R correlated with the ALS CBS. Worsening disinhibition (FBI-ALS) did not correlate with ALSFRS-R, FVC, or disease duration. Conclusion. We 
did not detect cognitive change. Behavioral change was detected, and increased disinhibition was found among patients with abnormal baseline behavioral scores. Disinhibition changes did not correlate with disease duration or progression. Baseline behavioral problems were associated with advanced, rapidly progressive disease and study attrition.

\section{Introduction}

Patients with ALS may exhibit cognitive deficits, ranging from mild executive or language dysfunction to severe deficits consistent with dementia. For others, cognition may be relatively preserved yet behavioral symptoms are present, ranging from mild apathy to severe behavioral dysfunction reflecting frontotemporal dementia (FTD). ALS patients may demonstrate cognitive and behavioral deficits simultaneously, and those with marked deficits in both domains typically suffer from ALS-FTD.

ALS patients with concomitant FTD have shorter survival [1]. Nondemented patients with mild-moderate cognitive or behavioral abnormalities are also impacted prognostically. Patients who present with any degree of executive dysfunction at the time of diagnosis are more likely to exhibit faster cognitive and motor progression [2]. ALS patients who are cognitively normal at baseline are more likely to remain cognitively unchanged over time. Behavioral changes may predate both motor and cognitive symptoms in ALS [3], although less is known about longitudinal behavioral change in nondemented ALS patients. The recent study [4] found that severe apathy is a significant negative prognostic indicator in ALS regardless of severity of physical disease.

We previously reported that among participants enrolled in ALS COSMOS, rates of cognitive and behavioral impairment were consistent with existing literature, which supports the utility of cognitive and behavioral screening tools in large trials [5]. Here, we report on longitudinal data from the ALS COSMOS study in order to describe the natural history of both cognition and behavior in ALS.

\section{Materials and Methods}

2.1. Participant Selection and Consent. The ALS COSMOS study is a large, prospective, multicenter, interdisciplinary, epidemiological study of oxidative stress which has been described previously [6]. Two hundred ninety-four participants were enrolled at baseline. Current data is based on 134 participants with both baseline and follow-up assessments. The mean time interval between assessments was $11.5 \pm 2.4$ months.

IRB approval was obtained at each clinical site. Written informed consent was provided by all participants and their caregivers.

2.2. Cognitive and Behavioral Screening. Participants repeated a number of neuropsychological screens of cognitive and behavioral functioning. The ALS Cognitive Behavioral Screen (ALS-CBS) [7], the Verbal Fluency Index C words (VFI) [8], and Controlled Oral Word Association Test (COWAT) [9] were used to assess executive functioning, attention, verbal fluency, and mental control. The behavioral scale of the ALS CBS was used to evaluate for changes in personality, social comportment, and apathy based on caregiver's report. The Frontal Behavioral InventoryALS (FBI-ALS) version [10] was also used, which is an interview-based tool. The FBI-ALS is comprised of the negative scale (measures apathy, emotional flatness, and aspontaneity) and the disinhibition scale (measures behaviors like inappropriateness, impulsivity, and hyperorality). The Center for Neurological Study-Lability Scale (CNS-LS) [11], completed by the patient, is a screening tool for pseudobulbar affect (PBA). For further explanation of study measures, please refer to the baseline study [5].

2.3. Clinical and Demographic Variables. Functional disability was measured using the ALS Functional Rating ScaleRevised (ALS FRS-R) [12], and ventilatory status was measured using forced vital capacity (FVC). Duration of symptoms prior to diagnosis, time since diagnosis, and site of onset were recorded. Illness onset was defined as the first sign of muscle weakness and/or symptoms caused by muscle weakness [13]. Fasciculations, cramps, and fatigue were not considered signs of illness onset. The MMSE (Mini-Mental State Examination) [14] was administered at baseline but not at follow-up. Demographic variables included age, gender, education, and ethnicity. All data were stored in the Data Management Center at the Columbia University Medical Center [6].

2.4. Statistics. Statistical analyses were conducted using SPSS version 23.0 and SAS version 9.4. We identified a set of covariates that were associated with baseline ALS functional measures for all analyses: age, sex, duration of symptoms, education, race, and ethnicity. Analysis of covariance (ANCOVA) procedures were used to compare demographic and illness characteristics of cases with only baseline assessments versus cases with baseline and followup data. Visual inspection of histograms and KolmogorovSmirnov tests were used to assess the normality assumption of ANCOVA.

Follow-up cognitive assessments were performed 5-18 months after baseline (mean: 11.5 months). We calculated the time between the two assessments and categorized this time into quartiles. We examined all cognitive and behavioral measures by these quartiles and found no differences. Because there were no differences in cognitive and behavioral change related to the time between assessments, all cases were subsequently examined together.

Generalized estimating equations (GEE) were used to evaluate change over time for all measures, controlling for the a priori covariates. Multiple regression analysis was conducted to further examine changes over time. Change scores were calculated for cognitive, behavioral, and disease progression measures by comparing baseline scores to scores at follow-up. Negative change score values indicate a worsening of condition from baseline to 12-month follow-up. 
TABLE 1: Comparative analysis of baseline data for two groups: 134 cases with follow-up data compared to 160 cases without follow-up data.

\begin{tabular}{|c|c|c|c|c|c|}
\hline Variable & $\begin{array}{l}\text { Participants completing baseline and } \\
\text { follow-up assessments (mean/SD) }\end{array}$ & $N$ & $\begin{array}{l}\text { Participants completing baseline } \\
\text { assessment only (mean/SD) }\end{array}$ & $N$ & $p$ \\
\hline$\overline{\text { Age }}$ & $59.7(10.1)$ & 134 & $61.8(9.9)$ & 160 & 0.076 \\
\hline Onset to baseline assessment & $14.3(4.9)$ & 134 & $12.8(4.9)$ & 160 & 0.012 \\
\hline Time from illness to enrollment & $12.6(4.4)$ & 134 & $11.1(4.3)$ & 160 & 0.005 \\
\hline ALSFRS-R & $38.5(5.0)$ & 129 & $34.2(7.1)$ & 154 & $<0.001$ \\
\hline FVC & $86.6(20.5)$ & 129 & $73.3(22.4)$ & 140 & $<0.001$ \\
\hline$\%$ male & 59.3 & 134 & 59 & 160 & 0.958 \\
\hline Race, \% white & 88.2 & 134 & 91.0 & 160 & 0.427 \\
\hline Hispanic ethnicity & 9.3 & 134 & 4.5 & 160 & 0.108 \\
\hline Education & & 134 & & 160 & \\
\hline$\% \mathrm{HS} / \mathrm{GED}$ & $26.7 \%$ & & $20.9 \%$ & & \\
\hline Some college, AA & $34.8 \%$ & & $29.1 \%$ & & \\
\hline $\mathrm{BA}, \mathrm{BS}$, or higher & $38.5 \%$ & & $50.0 \%$ & & 0.138 \\
\hline MMSE & $29.1(1.4)$ & 120 & $28.8(2.0)$ & 132 & 0.121 \\
\hline CBS cognitive score & $15.6(2.8)$ & 120 & $15.1(3.3)$ & 134 & 0.126 \\
\hline CBS behavioral score & $37.4(5.2)$ & 118 & $34.7(6.9)$ & 131 & 0.036 \\
\hline FBI negative scale & $3.2(4.9)$ & 119 & $4.0(4.7)$ & 132 & 0.119 \\
\hline FBI disinhibition scale & $1.45(2.56)$ & 119 & $1.87(2.31)$ & 132 & 0.127 \\
\hline CNS-Lability Scale & $12.2(4.5)$ & 130 & $13.7(5.4)$ & 158 & 0.009 \\
\hline COWAT total & $36.7(10.7)$ & 64 & $33.3(11.6)$ & 80 & 0.076 \\
\hline VFI C words & $13.8(7.7)$ & 77 & $11.3(6.9)$ & 52 & 0.177 \\
\hline
\end{tabular}

Change scores served as the dependent variable, while independent variables included the a priori covariates and the baseline score. Because the ALS CBS behavioral scores differed significantly from baseline to follow-up (decrement in function), we performed an item analysis of this scale first looking at the unadjusted changes in responses, then using general linear models, and controlling for covariates.

Using diagnostic criteria established by Strong et al. [15], we created diagnostic subgroups (normal, mild impairment, and moderate impairment) using the baseline ALS CBS cognition score, the baseline Verbal Fluency Index (normal and impaired) and the baseline ALS CBS behavioral score. Change scores were calculated for each diagnostic subgroup on each measure to examine the longitudinal progression of patients based on these classifications. We then performed a one-way ANCOVA to examine change scores across each diagnostic subgroup to determine whether baseline cognitive/behavioral diagnostic classification is associated with the degree of cognitive or behavioral change, if any. When significant differences were detected between diagnostic groups, we ran post hoc pairwise comparisons to determine specifically which groups differed from each other.

All tests were two-tailed, and significance was set at $p<0.05$ for analyses. We performed sensitivity analyses using Bonferroni-corrected alpha levels to control for multiple comparisons.

\section{Results}

3.1. Comparison of Cases with and without Cognitivel Behavioral Follow-Up Data. Cases with follow-up cognitive/ behavioral test scores had higher baseline ALSFRS-R $(p<0.001)$ and FVC $(p<0.001)$ ratings (Table 1$)$, compared to cases without follow-up data. Cases in the follow-up group also had longer disease durations at baseline $(p=0.012)$ and were two years younger than those without follow-up $(p=0.047)$, although this age difference was no longer significant after Bonferroni correction. Other demographic variables did not differ significantly between groups.

Further, cases with follow-up data had significantly higher scores (reflecting fewer behavior problems) on the baseline ALS-CBS behavioral scale total compared to cases with no follow-up data $(p=0.036)$. No differences were found between cases with and without follow-up data on the other cognitive and behavioral tests (Table 1).

3.2. Cognitive Impairment at Follow-Up. Based on the ALS CBS cognitive score at follow-up, 5.2\% of participants were classified in the range consistent with dementia and $54 \%$ in the mild cognitive impairment range (ALSci). This is consistent with baseline rates of impairment from the larger cohort (6.5\% and 54.2\%, respectively) [5]. Using the Verbal Fluency Index, $43.4 \%$ of the participants scored in the mildly impaired range at follow-up.

The cognitive screening tools (ALS CBS cognitive scale and VFI) did not reveal significant change during the follow-up period (Table 2). Although the sample means on the ALS CBS cognitive scale did not change over time (15.6 versus 15.4), regression analysis revealed that greater decline in performance was associated with being male $(p=0.034)$, longer duration between assessments $(p=0.033)$, and higher 
TABLE 2: Change over time: comparison of baseline and follow-up scores in 134 participants with longitudinal data, using GLM-GEE procedures, controlling for age, sex, duration of symptoms, education, race, and ethnicity (adjusted means and standard errors).

\begin{tabular}{|c|c|c|c|c|}
\hline & \multirow{2}{*}{ Baseline mean (SE) } & \multirow{2}{*}{ Follow-up mean (SE) } & \multicolumn{2}{|c|}{ Fixed effects for interval } \\
\hline & & & Wald chi-square value & $p$ value \\
\hline ALSFRS & $38.5(39.1)$ & $27.6(39.1)$ & 240.27 & $<0.001^{\perp}$ \\
\hline $\mathrm{FVC} \%$ & $86.7(95.0)$ & $64.5(94.7)$ & 114.13 & $<0.001^{\perp}$ \\
\hline ALS CBS cognitive score & $15.6(9.8)$ & $15.4(9.8)$ & 0.82 & 0.450 \\
\hline ALS CBS behavioral score & $37.5(31.0)$ & $35.5^{*}(30.9)$ & 15.16 & $<0.001^{\perp}$ \\
\hline Verbal Fluency Index ( $z$-score converted) & $-0.159(3.79)$ & $0.063^{* *}(3.74)$ & 1.51 & 0.252 \\
\hline FBI negative scale & $3.2(20.3)$ & $4.1(20.2)$ & 2.63 & 0.090 \\
\hline FBI disinhibition & $1.46(13.3)$ & $2.01(13.2)$ & 4.07 & 0.044 \\
\hline CNS lability score & $12.2(20.2)$ & $11.9^{* * *}(20.2)$ & 0.78 & 0.378 \\
\hline
\end{tabular}

${ }^{*}$ Decline in CBS behavioral score correlates with ALSFRS-R $(p=0.001) .{ }^{* *}$ Decline in VFI score correlates with ALSFRS-R $(p=0.010) .{ }^{* * *}$ Decline in CNS-LS score correlates with ALSFRS-R $(p=0.006)$ and FVC $(p=0.040) .{ }^{\perp}$ Significant at the Bonferroni-adjusted alpha level of 0.007 .

baseline CBS scores $(p<0.001)$. For sensitivity, we compared these $p$ values to the Bonferroni-corrected alpha level of 0.005 , for the 10 variables in the model. Higher baseline CBS scores persisted as a significant predictor of ALS CBS cognitive scale change using this conservative method. VFI decline was significantly associated with greater disease progression, as assessed by ALSFRS-R $(p=0.010)$, although fewer participants completed this screen at follow-up. The ALS CBS cognitive scale and COWAT did not associate with ALSFRS-R scores.

3.3. Behavioral Impairment at Follow-Up. Using ALS CBS behavioral cutoff scores [3], 14.9\% of the sample scored within the range that suggests dementia at follow-up. This is equivalent with the percentage of patients in the baseline cohort with probable dementia at baseline (16.5\%) [5]. Approximately $11.2 \%$ of participants scored within the range of mild behavioral impairment (ALSbi) at follow-up, comparable to $14.1 \%$ who were mildly impaired at baseline.

The ALS CBS behavioral total $(p<0.001)$ and the FBIALS disinhibition scale $(p=0.044)$ changed significantly during the follow-up period, suggesting behavioral deterioration, although the change in FBI-ALS disinhibition scale is no longer significant after Bonferroni correction (Table 2). Regression analysis showed that the decline in CBS behavior score was associated with disease progression (ALSFRS-R, $p=0.001)$. FBI disinhibition scores were not associated with any of the independent variables. The FBI-ALS negative scale remained stable from baseline to follow-up. Although the CNS-Lability Scale (CNS-LS) remained stable from baseline to follow-up, change on this measure was associated with disease progression (ALSFRS-R, $p=0.006$ and FVC, $p=0.040$ ) and the CNS-LS baseline score $(p=0.001)$.

We examined each item of the ALS CBS behavioral scale for change during follow-up. Without controlling for covariates, specific item scores that changed across the interval, reflecting deterioration in behavior, included the following: decreased interest in topics/events that used to be important, decreased ability to deal with frustration or stress, difficulty changing opinions/adapting to new situations, and decreased awareness of obvious problems/changes or denial. After adjusting for the same a priori covariates, all of these items except for "decreased interest in topics/events that used to be important," were significantly different with $p<0.0001$.

3.4. Change across Diagnostic Subgroups. Participants were classified into diagnostic subgroups (no impairment, mild impairment, and suspected dementia) according to consensus criteria [15] based on baseline ALS CBS scores. The ALS CBS cognitive change score differed among the three cognitive diagnostic subgroups (Table $3, p=<0.001$ ). Participants in the no impairment and mild cognitive impairment subgroups had relatively stable cognitive scores between baseline and follow-up. Surprisingly, the dementia subgroup showed improvement on the CBS cognitive total (Table 3). No changes on other cognitive measures were found among baseline diagnostic subgroups.

Similar analyses were completed across behavioral diagnostic subgroups based on the baseline ALS CBS behavioral score. We detected significant decline $(p=0.008)$ between baseline and follow-up on the FBI disinhibition scale for all three behavioral diagnostic subgroups (Table 4). The greatest decline in disinhibition was found among participants classified in the dementia subgroup at baseline compared to the nonimpaired and participants with mild behavioral impairment ( $p=0.002$ and 0.056 , respectively).

There were 6 cases identified as normal at baseline who transitioned to dementia at follow-up, exhibiting an expected and significant decline in behavioral scores $(p=0.002)$. However, these specific cases did not demonstrate an equivalent decline in ALS CBS cognitive scores $(p=0.320)$.

3.5. Site of ALS Onset. We examined all of the change scores with respect to the site of ALS onset (bulbar versus spinal), controlling for the a priori covariates. Bulbar-onset participants exhibited a greater decline in VFI $(p=0.013)$ compared to spinal-onset participants. No other change was evident.

\section{Discussion}

Significant behavioral change occurs in ALS patients over time, as indicated on screening measures completed by caregivers, including the FBI-ALS and ALS CBS. Behavioral change was not associated with disease duration or 
TABLE 3: Change in scores over time across diagnostic groups, defined by baseline ALS-CBS cognitive scores, controlling for age, sex, time from illness onset to baseline testing, and time between assessments (GLM ANCOVA, adjusted means, and standard errors are presented).

\begin{tabular}{|c|c|c|c|c|c|c|c|c|c|}
\hline \multicolumn{10}{|c|}{ Change scores across diagnostic subgroups (cognitive)* } \\
\hline \multirow{3}{*}{ Cognitive and behavioral screens } & \multirow{2}{*}{\multicolumn{2}{|c|}{$\begin{array}{c}1 \\
\text { Normal } \\
N=48-49\end{array}$}} & \multirow{2}{*}{\multicolumn{2}{|c|}{$\begin{array}{c}2 \\
\text { Mild } \\
N=60-62\end{array}$}} & \multirow{2}{*}{\multicolumn{2}{|c|}{$\begin{array}{c}3 \\
\text { Moderate } \\
N=7-9\end{array}$}} & \multirow{2}{*}{\multicolumn{2}{|c|}{ ANCOVA }} & \multirow{2}{*}{ Pairwise } \\
\hline & & & & & & & & & \\
\hline & $\mathrm{MN}$ & SE & $\mathrm{MN}$ & SE & $\mathrm{MN}$ & SE & $F$ & $p$ & $p<0.05$ \\
\hline ALS-CBS cognition & -1.12 & 0.373 & 0.13 & 0.335 & 4.33 & 0.983 & 13.92 & $0.001^{* \perp}$ & All \\
\hline ALS-CBS behavior & -1.21 & 0.820 & -2.50 & 0.730 & -3.12 & 1.88 & 0.85 & 0.430 & None \\
\hline FBI negative & -0.89 & 0.82 & -0.92 & 0.73 & -.61 & 1.90 & 0.01 & 0.989 & None \\
\hline FBI disinhibition & -0.32 & 0.39 & -0.77 & 0.35 & -2.48 & 0.90 & 2.45 & 0.091 & 1 vs. 3 \\
\hline CNS-Lability Scale & -0.27 & 0.56 & 0.66 & 0.50 & 0.67 & 1.30 & 0.78 & 0.460 & None \\
\hline
\end{tabular}

*Diagnostic subgroups were determined based on baseline ALS CBS scores on the cognitive screen [7]. Normal: no significant cognitive impairment; mild: suspicion of mild cognitive impairment; moderate: suspicion of moderate-severe cognitive impairment, raising suspicion for dementia. ${ }^{\perp}$ Significant at the Bonferroni-adjusted alpha level of 0.01 .

TABLE 4: Change in scores over time across diagnostic groups, defined by baseline ALS-CBS behavioral scores, controlling for age, sex, time from illness onset to baseline testing, and time between assessments (GLM ANCOVA, adjusted means, and standard errors are presented).

\begin{tabular}{|c|c|c|c|c|c|c|c|c|c|}
\hline \multicolumn{10}{|c|}{ Change scores across diagnostic subgroups (behavioral)* } \\
\hline \multirow{3}{*}{ Cognitive and behavioral screens } & \multirow{2}{*}{\multicolumn{2}{|c|}{$\begin{array}{c}1 \\
\text { Normal } \\
N=86-93\end{array}$}} & \multirow{2}{*}{\multicolumn{2}{|c|}{$\begin{array}{c}2 \\
\text { Mild } \\
N=10-11\end{array}$}} & \multirow{2}{*}{\multicolumn{2}{|c|}{$\begin{array}{c}3 \\
\text { Moderate } \\
N=13-16\end{array}$}} & \multirow{2}{*}{\multicolumn{2}{|c|}{ ANCOVA }} & \multirow{2}{*}{ Pairwise } \\
\hline & & & & & & & & & \\
\hline & $\mathrm{MN}$ & $\mathrm{SE}$ & $\mathrm{MN}$ & SE & $\mathrm{MN}$ & SE & $F$ & $p$ & $p<0.050$ \\
\hline ALS-CBS cognition & -0.23 & 0.313 & -0.31 & 0.917 & 0.46 & 0.823 & 0.32 & 0.729 & None \\
\hline ALS-CBS behavior & -2.21 & 0.60 & -3.00 & 1.71 & -0.26 & 1.47 & 0.93 & 0.400 & None \\
\hline FBI negative & -0.79 & 0.59 & -0.17 & 1.71 & -1.96 & 1.52 & 0.35 & 0.708 & None \\
\hline FBI disinhibition & -0.37 & 0.274 & -0.76 & 0.793 & -2.80 & 0.706 & 5.01 & $0.008^{* \perp}$ & 1 vs. 3 \\
\hline CNS-Lability Scale & 0.46 & 0.42 & -0.55 & 1.18 & -0.22 & 1.02 & 0.45 & 0.640 & None \\
\hline
\end{tabular}

${ }^{*}$ Diagnostic subgroups were determined based on baseline ALS CBS scores on the behavioral screen [7]. Normal: no significant behavioral impairment; mild: suspicion of mild behavioral impairment; moderate: suspicion of moderate-severe behavioral impairment, raising suspicion for dementia. ${ }^{\perp}$ Significant at Bonferroni-adjusted alpha level of 0.01 .

respiratory decline, but changes detected with the ALS CBS were associated with functional disability (ALSFRS-R scores). In contrast, disinhibition measured by the FBI-ALS was not associated with any disease severity indices.

Participants with behavioral problems consistent with FTD at baseline demonstrated more rapid progression of behavioral disinhibition over time compared to behaviorally normal participants or those with mild behavioral abnormalities. Despite the high prevalence of apathy in ALS $[16,17]$, significant change in this behavioral symptom was not detected using the FBI negative scale. However, an item analysis of the ALS CBS suggested progression of apathy, identified by change on an item measuring interest in topics and activities. Other behavioral symptoms that changed significantly over time included reduced frustration tolerance, reduced adaptability to new situations/changing opinions, and decreased insight. These results contribute to the existing literature that suggests that subtle behavioral changes are pervasive in ALS [3] and may be seen in patients without a clinically obvious syndrome [18].

Behavioral data was obtained from caregivers via a written questionnaire (ALS CBS) and interview (FBI-ALS). Caregiver perspectives can be biased by factors such as disease severity, mood, and psychosocial stress, which may lead to measurement error. The recent study found that behavioral impairment, but not cognitive impairment, negatively impacted caregiver burden in ALS [19]. Future behavioral studies may be strengthened by controlling for caregiver burden and caregiver depression/anxiety [20], which may provide an alternative explanation for increased rates of reported behavioral abnormalities over time and correlation with functional disability.

Using ALS CBS behavioral score cutoffs [7], nearly $15 \%$ of the sample fell within the range suggestive of FTD at follow-up. When FTD was estimated based on cognition rather than behavior, the rate was only $5.2 \%$. It is possible that the rate discrepancy reflects the behavioral basis of FTD, since initial FTD symptoms frequently include social deficits or personality change rather than overt problems with concentration, set shifting, or fluency. Cognitive screening, if used without behavioral data, may underestimate the rate of dementia in ALS research cohorts.

Cognitive decline was not detected in this study. Study attrition correlates with cognitive impairment in ALS [2], and longitudinal studies may be enriched with cognitively intact patients [2]. Our results did not replicate this 
association, but we did detect an association between behavioral impairment and attrition. Participants who did not remain in the study were older and had more advanced disease that progressed more rapidly. These participants also had more baseline behavioral problems, suggesting an association between behavioral symptoms and more aggressive motor disease. Caga et al. [4] studied apathy and found that participants with the most severe behavioral impairment have significantly shorter mean survival (22 months) compared to those with mild apathy (46.9 months) or those without apathy (52 months).

Analysis of the baseline data from this cohort [5] suggested a significant correlation between apathy and cognitive impairment. These data also suggested a correlation between the absence of cognitive impairment and the presence of behavioral disinhibition. When considered within the context of attrition and longitudinal follow-up, the behavioral data makes sense. If longitudinal trials enrich for cognitively intact participants [2], then we may expect to see more disinhibition over time since it correlates with a relative lack of cognitive impairment. In contrast, since apathy correlated with cognitive impairment at baseline, we do not see marked increases in apathy over time because cognitively impaired patients may have a greater risk of attrition. These data suggest the possibility of two distinct phenotypes for further study: (1) patients with mild cognitive impairment and apathy and (2) cognitively normal patients with disinhibition.

\section{Data Availability}

This multicenter trial does not currently have IRB permission to share data. Permission was requested by the lead authors but the IRB had not granted approval by the time of publication.

\section{Disclosure}

Preliminary portions of these data were presented at the 2014 International MND Meeting.

\section{Conflicts of Interest}

Jennifer Murphy is employed by the CRO Syneos Health. Bjorn Oskarsson served as a speaker for Grifols. Sharon Nations serves as a consultant for Grifols.

\section{Authors' Contributions}

Susan Woolley is the primary author and was involved in data analysis and interpretation and all aspects of manuscript development and revision. Ray Goetz was involved with statistical analyses, data interpretation, and critical review of the manuscript. Pam Factor-Litvak served as a coinvestigator for this study and was involved in all stages of this project, including study design, planning, statistical analyses, data interpretation, and critical review and revision of the manuscript. Jennifer Murphy was involved in study planning, data interpretation, and review of the manuscript. Jonathan Hupf assisted in the planning and coordination of the study, data collection, and review of the manuscript. Catherine Lomen-Hoerth served as a site investigator for University of California, San Francisco, and was involved with study planning and data collection. Howard Andrews assisted in data management for the study. Daragh Heitzman served as a site investigator for Texas Neurology, Dallas, Texas, and was involved with study planning and data collection. Richard Bedlack served as a site investigator for Duke University and was involved with study planning and data collection. Jonathan Katz served as a site investigator for California Pacific Medical Center and was involved with study planning, data collection, and review of the manuscript. Richard Barohn served as a site investigator for University of Kansas and was involved with study planning, data collection, and review of the manuscript. Eric Sorenson served as a site investigator for Mayo Clinic, Rochester, was involved with study planning and data collection, and served on the study's publication committee. Bjorn Oskarsson served as a site investigator for University of California, Davis, was involved with study planning and data collection, and served on the study's publication committee. Americo Fernandes Filho served as a site investigator for University of Nebraska, was involved with study planning and data collection, and served on the study's publication committee. Edward Kasarskis served as a site investigator for University of Kentucky and was involved with study planning, data collection, and review of the manuscript. Tahseen Mozaffar served as a site investigator for University of California, Irvine, and was involved with study planning, data collection, and review of the manuscript. Sharon Nations served as a site investigator for University of Texas, Southwestern, and was involved with study planning and data collection. Andrea Swenson served as a site investigator for University of Iowa Hospitals and Clinics and was involved with study planning and data collection. Agnes Koczon-Jaremko served as a site investigator for Hospital for Special Care, New Britain, Connecticut, and was involved with study planning and data collection. Georgia Christodoulou assisted on the study and was involved in formatting and editing of the manuscript. Hiroshi Mitsumoto served as the primary investigator for this study and was involved in all stages of this project, including study design, data collection, statistical analyses, and review of the manuscript.

\section{Acknowledgments}

The authors acknowledged B. A. Jessica Singleton who was a research assistant during part of the ALS COSMOS study. We thank the many patients and families who participated in this study and the ALS COSMOS Study Group site investigators. The authors also acknowledged Ray Goetz, PhD, Department of Psychiatry, New York State Psychiatric Institute, Columbia University Medical Center, who died in December 2017, for conducting the statistical analysis. Pam Factor-Litvak received travel funds from the Environment and Health Fund and travel funds and honorarium to serve as a reviewer for the European Union 2020 grant initiative. Daragh Heitzman received financial support from the 
Muscular Dystrophy Association and research support from National Institute of Health (via Columbia University Subcontract), Biogen Idec, Questcor Pharmaceuticals, Merz Pharmaceuticals, and Cytokinetics for research trials as a site investigator. Richard Bedlack received consulting support from ALSA, Neuraltus, Ultragenyx, Pinnacle Research Group, Lighthouse Research Group, and Guidepoint Global and research support from Amyotrophic Lateral Sclerosis Association, Motor Neurone Disease Association, Iron Horse Diagnostics, Cytokinetics, Neuraltus, and GlaxoSmithKline. Bjorn Oskarsson received research support from NIH, Amyotrophic Lateral Sclerosis Association, Novartis, BMS, and Cytokinetics. Tahseen Mozaffar received personal compensation from consulting activities to Baxter, Biogen Idec, BioMarin, California Stem Cell Inc., Crescent (a Walgreens company) CSL Behring, Genzyme, Grifols, Idera, NuFACTOR, and Ultragenyx. Dr. Mozaffar received funding from NIH (\#NS049203) and received clinical research support from ALS Therapy Development Institute, Alexion Pharmaceuticals, Alnylam Pharmaceuticals, Amicus, Biogen Idec, BioMarin Pharmaceutical, CSL Behring, Cytokinetics, Food and Drug Administration, Grifols, Genzyme, GlaxoSmithKline, ISIS, Neuraltus, Novartis, and Ultragenyx Pharmaceutical. Hiroshi Mitsumoto received government research support from NIEHS (R01ES016348) and research support from MDA Wings Over Wall Street for the publication. This study was funded by NIEHS (R01ES016348), MDA Wings Over Wall Street, and Anthony Senerchia Jr. ALS Charitable Foundation.

\section{References}

[1] M. Elamin, J. Phukan, P. Bede et al., "Executive dysfunction is a negative prognostic indicator in patients with ALS without dementia," Neurology, vol. 76, no. 14, pp. 1263-1269, 2011.

[2] M. Elamin, P. Bede, S. Byrne et al., "Cognitive changes predict functional decline in ALS: a population-based longitudinal study," Neurology, vol. 80, no. 17, pp. 1590-1597, 2013.

[3] E. Mioshi, J. Caga, P. Lillo et al., "Neuropsychiatric changes precede classic motor symptoms in ALS and do not affect survival," Neurology, vol. 82, no. 2, pp. 149-155, 2014.

[4] J. Caga, M. R. Turner, S. Hsieh et al., "Apathy is associated with poor prognosis in amyotrophic lateral sclerosis," European Journal of Neurology, vol. 23, no. 5, pp. 891-897, 2016.

[5] J. Murphy, P. Factor-Litvak, R. Goetz et al., "Cognitivebehavioral screening reveals prevalent impairment in a large multicenter ALS cohort," Neurology, vol. 86, no. 9, pp. 813$820,2016$.

[6] H. Mitsumoto, P. Factor-Litvak, H. Andrews et al., "ALS Multicenter Cohort Study of Oxidative Stress (ALS COSMOS): study methodology, recruitment, and baseline demographic and disease characteristics," Amyotrophic Lateral Sclerosis and Frontotemporal Degeneration, vol. 15, no. 3-4, pp. 192203, 2014.

[7] S. C. Woolley, M. K. York, D. H. Moore et al., "Detecting frontotemporal dysfunction in ALS: utility of the ALS Cognitive Behavioral Screen (ALS-CBS ${ }^{\mathrm{TM}}$ )," Amyotrophic Lateral Sclerosis, vol. 11, no. 3, pp. 303-311, 2010.

[8] S. Abrahams, P. N. Leigh, A. Harvey, G. N. Vythelingum, D. Grise, and L. H. Goldstein, "Verbal fluency and executive dysfunction in amyotrophic lateral sclerosis (ALS)," Neuropsychologia, vol. 38, no. 6, pp. 734-747, 2000.

[9] R. Reitan, Halstead-Reitan Neuropsychological Test Battery: Theory and Clinical Interpretation, Reitan Neuropsychology, Tucson, Arizona, 1985.

[10] J. Murphy, F. Ahmed, and C. Lomen-Hoerth, "The UCSF screening exam effectively screens cognitive and behavioral impairment in patients with ALS," Amyotrophic Lateral Sclerosis and Frontotemporal Degeneration, vol. 16, no. 1-2, pp. 2430, 2015.

[11] S. R. Moore, L. S. Gresham, M. B. Bromberg, E. J. Kasarkis, and R. A. Smith, "A self report measure of affective lability," Journal of Neurology, Neurosurgery, and Psychiatry, vol. 63, no. 1, pp. 89-93, 1997.

[12] J. M. Cedarbaum, N. Stambler, E. Malta et al., "The ALSFRS-R: a revised ALS functional rating scale that incorporates assessments of respiratory function," Journal of the Neurological Sciences, vol. 169, no. 1-2, pp. 13-21, 1999.

[13] B. R. Brooks, R. G. Miller, M. Swash, and T. L. Munsat, "El Escorial revisited: revised criteria for the diagnosis of amyotrophic lateral sclerosis," Amyotrophic Lateral Sclerosis and Other Motor Neuron Disorders, vol. 1, no. 5, pp. 293-299, 2000.

[14] M. F. Folstein, S. E. Folstein, and P. R. McHugh, "Mini-mental state: a practical method for grading the cognitive state of patients for the clinician," Journal of Psychiatric Research, vol. 12, no. 3, pp. 189-198, 1975.

[15] M. J. Strong, G. M. Grace, M. Freedman et al., "Consensus criteria for the diagnosis of frontotemporal cognitive and behavioural syndromes in amyotrophic lateral sclerosis," Amyotrophic Lateral Sclerosis, vol. 10, no. 3, pp. 131146, 2009.

[16] E. Mioshi, S. Hsieh, J. Caga et al., "A novel tool to detect behavioural symptoms in ALS," Amyotrophic Lateral Sclerosis and Frontotemporal Degeneration, vol. 15, no. 3-4, pp. 298304, 2014.

[17] P. Lillo, E. Mioshi, M. C. Zoing, M. C. Kiernan, and J. R. Hodges, "How common are behavioural changes in amyotrophic lateral sclerosis?," Amyotrophic Lateral Sclerosis, vol. 12, no. 1, pp. 45-51, 2011.

[18] P. Lillo, S. Savage, E. Mioshi, M. C. Kiernan, and J. R. Hodges, "Amyotrophic lateral sclerosis and frontotemporal dementia. A behavioural and cognitive continuum," Amyotrophic Lateral Sclerosis, vol. 13, no. 1, pp. 102-109, 2012.

[19] L. Tremolizzo, A. Pellegrini, E. Susani et al., "Behavioural but not cognitive impairment is a determinant of caregiver burden in amyotrophic lateral sclerosis," European Neurological Journal, vol. 75, no. 3-4, pp. 191-194, 2016.

[20] P. H. Gordon, Y. Wang, C. Doorish et al., "A screening assessment of cognitive impairment in patients with ALS," Amyotrophic Lateral Sclerosis, vol. 8, no. 6, pp. 362-365, 2007. 


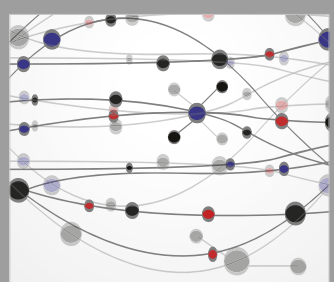

The Scientific World Journal
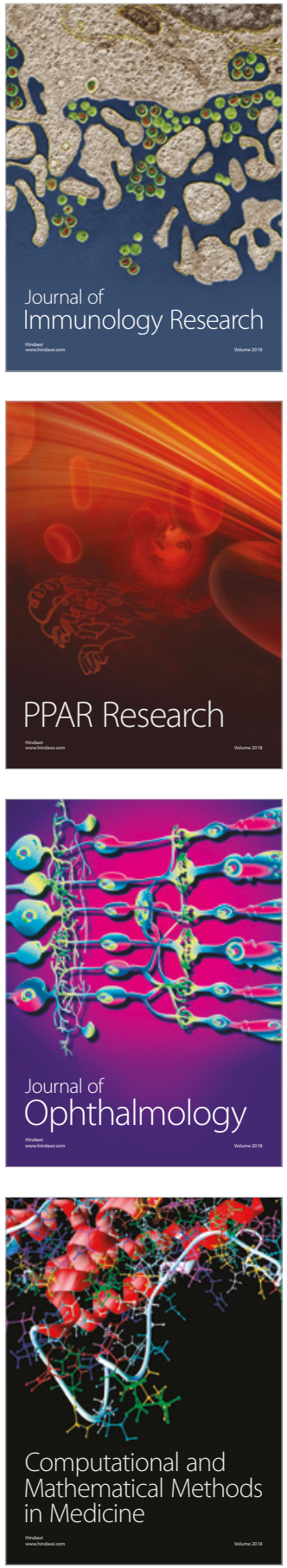

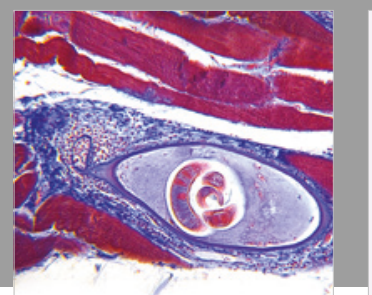

Gastroenterology Research and Practice

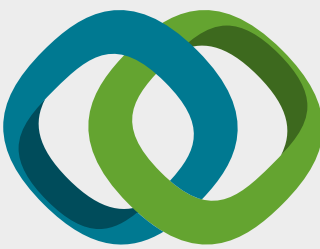

\section{Hindawi}

Submit your manuscripts at

www.hindawi.com
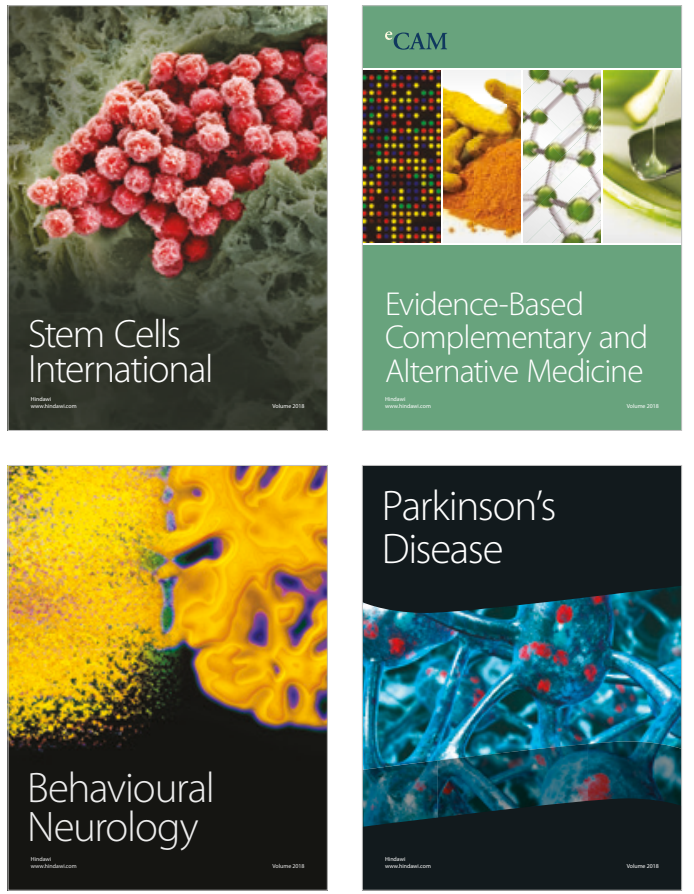

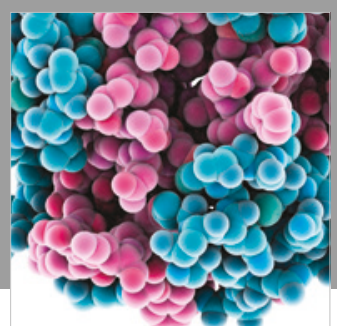

ournal of

Diabetes Research

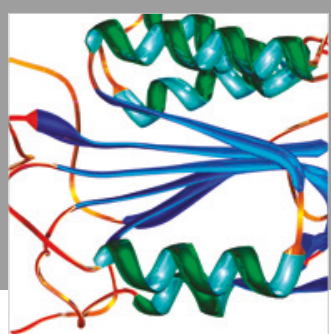

Disease Markers
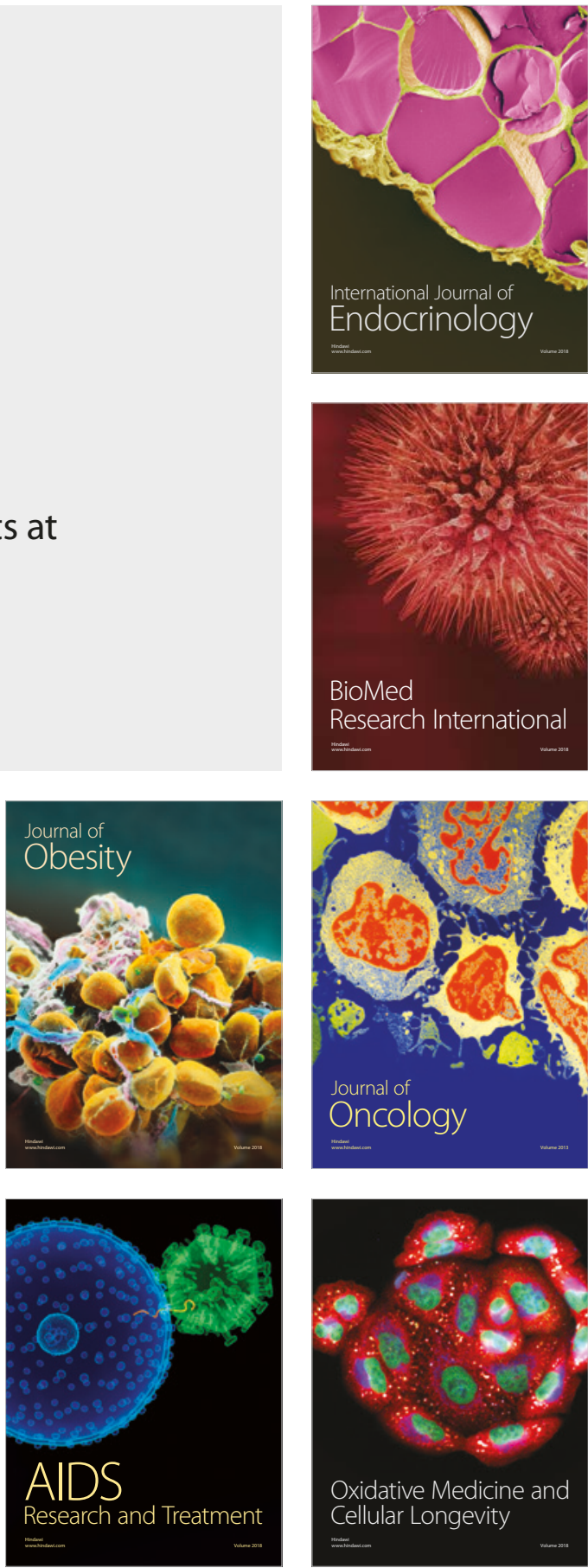